\title{
JUSTICIA DE GÉNERO EN EL PODER JUDICIAL DE COSTA RICA: UN ANÁLISIS DE POLÍTICA PÚBLICA
}

\section{GENDER JUSTICE IN THE JUDICIAL BRANCH OF COSTA RICA: A PUBLIC POLICIES ANALYSIS}

\author{
Andrea Bermúdez Castillo*
}

\section{RESUMEN}

El objetivo de este artículo es analizar, desde una perspectiva feminista, la Política Pública de Igualdad y Equidad de Género del Poder Judicial de Costa Rica, aprobada en noviembre del año 2005. El principal referente teórico es el paradigma de justicia de género, por lo tanto, de su aplicación se concluye que a pesar de los esfuerzos, persiste discriminación hacia las mujeres, tanto en el funcionamiento interno de la institución como a nivel de tutela de derechos y acceso a un "juicio justo", por lo cual es preciso formular una nueva política que responda a los desafíos actuales.

PALABRAS CLAVE: FEMINISMO * ADMINISTRACIÓN DE JUSTICIA * POLÍTICA PÚBLICA * DISCRIMINACIÓN SEXUAL * DERECHOS CIVILES

\section{ABSTRACT}

This article aims to analyze from a feminist perspective the Gender Equality and Equity Public Policy of the Judiciary Main Branch of Costa Rica approved in November 2005. The main theoretical reference is the paradigm of gender justice, therefore, its application concludes that, despite the efforts, discrimination against women persist, both in the internal functioning of the institution as well as at the level of protection of rights and access to a "fair trial". Hence, it is necessary to formulate a new policy that responds to current challenges.

KEYWORDS: FEMINISM * ADMINISTRATION OF JUSTICE * PUBLIC POLICY * GENDER DISCRIMINATION * CIVIL RIGHTS

Fiscalía Adjunta de Probidad, Transparencia y Anticorrupción, Costa Rica. solandrea28@yahoo.com / abermudezca@Poder-Judicial.go.cr 


\section{INTRODUCCIÓN}

La Política de Igualdad de Género del Poder Judicial (PIGPJ), aprobada por la Corte Plena en el año 2005, es una de las primeras políticas públicas implementadas en Costa Rica $y$ en la región centroamericana con la finalidad explícita de garantizar la igualdad de oportunidades entre mujeres $y$ hombres, así como, la no discriminación por género en las decisiones judiciales, en el servicio público de la administración de justicia $y$ en el funcionamiento institucional interno (Poder Judicial, 2005). Por lo tanto, representa un esfuerzo pionero en materia de acceso a la justicia para poblaciones vulnerables $y$ vulnerabilizadas.

Este escrito se dedica a su análisis desde una perspectiva feminista, tomando como referente metodológico la propuesta de Cristina Benavente y Alejandra Valdés (2014), que sustenta categorías desde el paradigma de la justicia de género para sistematizar el ciclo de las políticas públicas y ponderar su aporte en la deconstrucción de las relaciones de poder y la consolidación de mecanismos para la mejora de las condiciones de vida de las mujeres. El artículo se organiza en cuatro apartados: el primero presenta el marco conceptual, el segundo argumenta las implicaciones metodológicas, el tercero desarrolla las categorías sistematizando la información recopilada de documentos oficiales $y$ de 3 entrevistas semiestructuradas realizadas específicamente para este trabajo. Las conclusiones y recomendaciones se enuncian en la cuarta sección.

\section{JUSTICIA DE GÉNERO Y ACCESO A LA JUSTICIA EN LAS POLÍTICAS PÚBLICAS DEL PODER JUDICIAL}

El horizonte de la justicia de género como enfoque analítico es lograr igualdad $y$ un disfrute pleno de derechos de jure y de facto; por medio de acciones para compensar las desventajas de la subordinación de las mujeres, así como permitir acceso y control a los recursos que históricamente les han sido limitados o negados (Benavente y Valdés, 2014) por las relaciones estructurantes de la jerarquía del género. Esta sección reflexiona respecto a los fundamentos de este enfoque como referente para las políticas en el campo de interpretación y aplicación de las leyes, considerado un territorio en disputa para la igualdad de género (Facio, 1999).

El Estado y el derecho remiten a la idea de contrato social, es decir, al acuerdo por el cual quienes le integran pactan en condiciones de igualdad $y$ ceden el monopolio de la violencia legítima al Estado para que dirima los conflictos. Pero el pacto excluyó a las mujeres, negando su ciudadanía y apropiándose de la economía sexual doméstica que transfiere valor, y es invisibilizada por asumirse inherente a quienes se encuentran en su posición de género (Pateman, 1995). El campo jurídico conjunta instituciones y prácticas para crear e interpretar el derecho y hacerlo parte de las decisiones de la sociedad (Trubek y Dezalay, 1997) y tiene como característica la potestad de nombrar $y$ crear realidad autorizada, una visión del mundo de la que no puede sustraerse el resto de la sociedad (Bourdieu, 2002). En ese sentido, ha contribuido a legitimar el estatus de inequidad $y$ constituye una expresión de intereses de dominio racial, religioso, de clase y etnia (Carcedo, 2002); junto con estas condiciones, la diferencia sexual - socialmente dotada de contenido- persiste como uno de los mecanismos principales para la reproducción de las desigualdades, configurando la posición y el estatus.

Sin embargo, el derecho puede utilizarse, desde otros paradigmas, como herramienta de cambio social. Rita Laura Segato (2003) reflexiona respecto a la forma en que la ley puede contribuir a modificar las estructuras del sistema sexo-género, destaca la creación discursiva y nominal, así como, su incidencia en las representaciones del mundo $y$ en desnaturalizar el orden vigente. Alda Facio y Lorena Fries (2005) coinciden en estos planteamientos, en cuanto al valor simbólico de la ley, al estímulo o inhibición que supone para las conductas y al modelo de sociedad ideal que establece.

Desde el ámbito de los derechos humanos, la conciencia de la desigualdad de género constituye uno de los principales aportes del feminismo del siglo xx, que complejizó su pretendida universalidad y permitió visibilizar 
que además de la igualdad formal ante la ley, también es necesario el reconocimiento de la diferencia para lograr equidad en los estrados judiciales y en la vida social. El género es una condición que puede obstaculizar el acceso a la justicia, por el trato discriminatorio que reciben quienes se identifican desde una posición de género femenino o también quienes subvierten los mandatos hegemónicos de la heteronormatividad.

Para transformar las relaciones opresivas de género desde el ámbito jurídico, los cursos de acción que se encaminan a la concreción de un objetivo público definido en forma democrática (Benavente y Valdés, 2014) pueden enfocarse en promover, garantizar o ampliar el acceso a la justicia, es decir:

... el derecho fundamental que tiene toda persona para acudir $y$ promover la actividad de los órganos encargados de prestar servicio público de impartición de justicia, con la finalidad de obtener tutela jurídica de sus intereses a través de una resolución pronta, completa e imparcial (Reglas de Brasilia, 2008, p.10).

El acceso a la justicia alude a las dimensiones interrelacionadas que conforman el derecho como producto social. La dimensión normativa desde esta perspectiva (Birgin $y$ Gherardi, 2008) se refiere a la desigualdad escrita en "letra de ley", comprende códigos y disposiciones que se basan en estereotipos de género; también comprende las consecuencias negativas de una aplicación imparcial y poco equitativa de la ley. Se expresa en la justiciabilidad, es decir, en la capacidad de respuesta del sistema judicial ante la desigualdad de género (Comité CEDAW, 2015). Dos referentes que amplían el marco para el análisis de la igualdad formal son la Convención sobre la eliminación de todas las formas de discriminación contra la mujer (CEDAW) y la Convención Belem do Pará. La CEDAW fue suscrita por la Organización de Naciones Unidas en el año 1979 y establece la responsabilidad estatal de adoptar distintas medidas para garantizar la igualdad en todos ámbitos de la vida social.
Por su parte, la Convención Interamericana para Prevenir, Sancionar y Erradicar la Violencia contra la Mujer, adoptada por la Asamblea General de la Organización de los Estados Americanos en 1994, es la primera convención continental específica contra la violencia como manifestación de la desigualdad estructural. Dispone que "Toda mujer tiene derecho a una vida libre de violencia, tanto en el ámbito público como en el privado" (art. 3) y para el debido reconocimiento, ejercicio y protección de sus libertades debe garantizarse, según el art. 4 en su inciso f) "El derecho a igualdad de protección ante la ley y de la ley"; $y$ de acuerdo al inciso g) "El derecho a un recurso sencillo y rápido ante los tribunales competentes, que la ampare contra actos que violen sus derechos".

La justiciabilidad es más amplia que la igualdad formal, ya que introduce una serie de elementos: que los casos se manejen de una manera sensible al género, que se eliminen las barreras para que ellas estén presentes en los ámbitos de aplicación de la ley - tradicionalmente masculinizados - tal es el caso de juzgados, tribunales, fiscalías, cuerpos policiales, entre otros; así como revisar las normas sobre la carga de la prueba en los procesos, con el fin de equilibrar las relaciones entre las partes, lo cual entra en el terreno de las acciones afirmativas que procuran eliminar el desbalance estructural de poder (Comité CEDAW, 2015).

Junto con la dimensión normativa, el acceso a la justicia considera los hechos que rodean los procedimientos judiciales y comprende la posibilidad real de reclamo ante las instituciones (Birgin y Gherardi, 2008). Usualmente esta es la dimensión más compleja de abordar, pues existen discriminaciones de facto que se asientan en imaginarios sociales que prescriben ciertas normas de comportamiento como adecuadas y permitidas para cada uno de los géneros, incidiendo en la interpretación de las leyes $y$ en la actividad persecutoria, investigativa y gerencial que realizan las funcionarias $y$ los funcionarios judiciales.

Otro de los obstáculos para las mujeres tiene que ver con su desigualdad situada: se reconoce que existe una experiencia de género 
común que es su condición (Lagarde, 1998) pero que las formas específicas en que se expresa resultan de circunstancias vitales, en las que se combinan lo histórico y cultural. El género es una relación y una práctica, cuyo contenido lo construyen también otras relaciones como las de clase, éticas, territoriales, raciales, etarias, religiosas, heteronormativas (Castañeda, 2012); su confluencia incide en la posibilidad material, física y simbólica de recurrir a las instancias judiciales como denunciante o testigo, principalmente en aquellos delitos con un sesgo de género, por ejemplo, los de tipo sexual y los contemplados dentro de la Ley de Penalización de la Violencia Contra las Mujeres (LPVCM).

El empoderamiento y autonomía son aspectos medulares para el acceso a la justicia con enfoque de género, tal como lo señalan Benavente y Valdés (2014), son requisito para que la igualdad sea posible. El empoderamiento puede entenderse como una toma de conciencia personal sobre las relaciones jerárquicas entre los géneros y la necesidad de transformarlas; mientras que la autonomía alude al grado de libertad que posee una mujer y que le posibilita elegir y actuar de acuerdo con su propio criterio. La justicia de género debe articularse en torno a estos dos ejes, pues en la medida en que se afirmen, pueden las mujeres constituirse en actoras centrales y no ser únicamente destinatarias de la acción del Estado.

\section{METODOLOGÍA DEL ESTUDIO}

Desde la definición de Lahera (2002), las políticas públicas se entienden como cursos de acción y flujos de información dinámicos desarrollados por el sector público, con participación de la comunidad y del sector privado, dirigidos a un objetivo definido democráticamente que afecta a la colectividad. Desde la perspectiva de Benavente y Valdés (2014), las políticas públicas establecen una obligación de los Estados y son exigibles por parte de la ciudadanía al ostentar un carácter institucional. En razón de lo anterior, son materia privilegiada de evaluación, sin embargo, el presente ejercicio no busca evaluar, pues hacerlo implica un abordaje teórico metodológico distinto y exhaustivo que supera lo aquí propuesto.

El alcance de este trabajo es registrar avances $y$ dificultades que en la práctica ha tenido la Política de Igualdad de Género del Poder Judicial, que constituye una iniciativa estatal orientada a garantizar los derechos de las mujeres. Para desarrollar el análisis, se utilizó como principal referente la matriz construida por María Cristina Benavente y Alejandra Valdés (2014) para el Observatorio de Igualdad de Género de América Latina y el Caribe, este instrumento operacionaliza el enfoque de justicia de género aplicado específicamente al estudio de políticas públicas y fue concebido como una herramienta rigurosa, resultado del debate experto en su diseño, validación y aplicación (Benavente y Valdés, 2014).

Una de sus fortalezas metodológicas es que contempla las distintas fases del ciclo de las políticas públicas: identificación y definición de los problemas, formulación, implementación, seguimiento y evaluación, lo que facilita un estudio sistemático, mostrando la complejidad de las políticas y su capacidad para transformar las injusticias en contextos específicos, considerando criterios de justicia distributiva, de reconocimiento y de representación. Las variables analíticas de la matriz se resumen en el cuadro 1. 


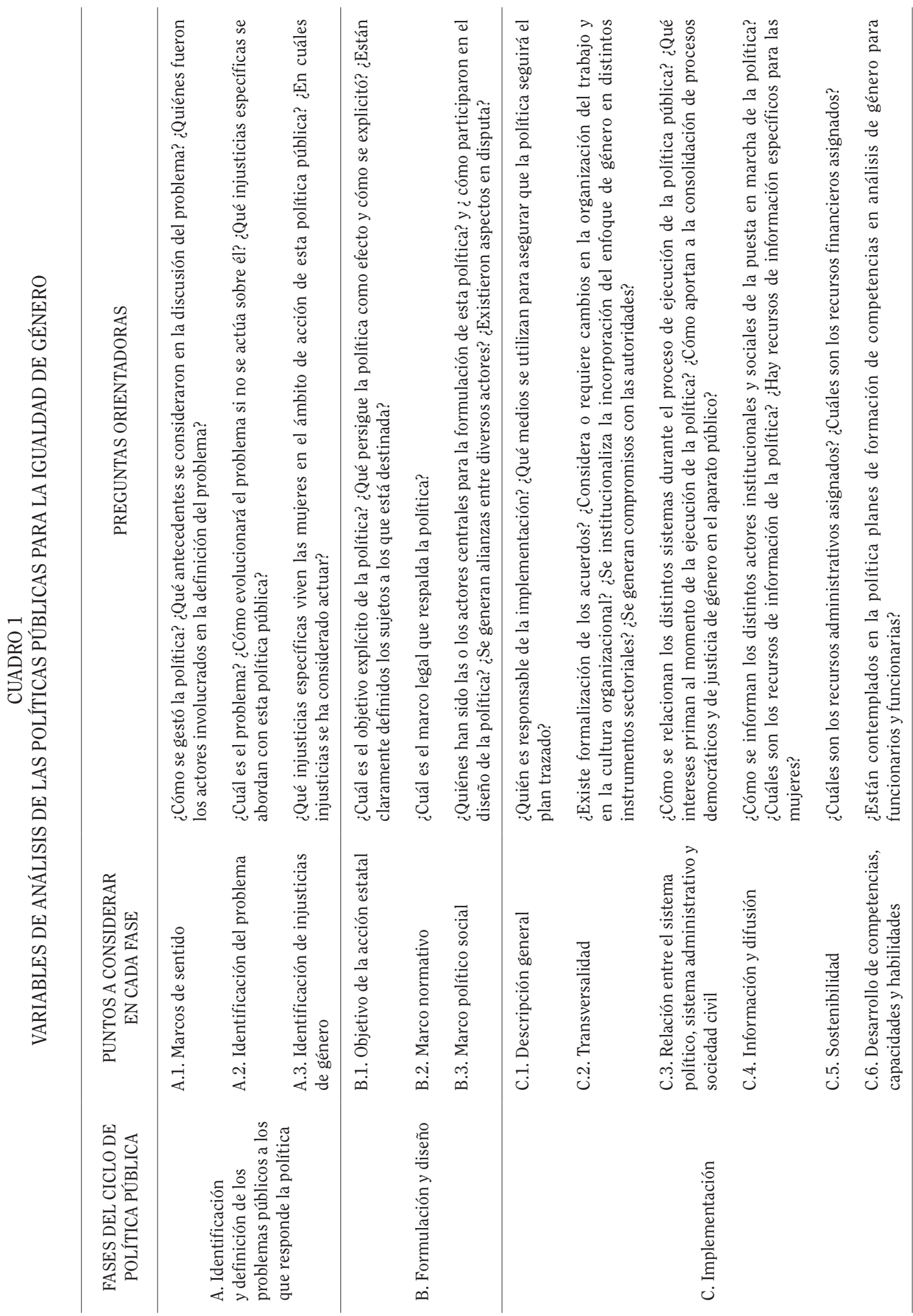

Rev. Ciencias Sociales Universidad de Costa Rica, 165: 135-154 / 2019 (III). (ISSN: 0482-5276) (C) (7) (8) 


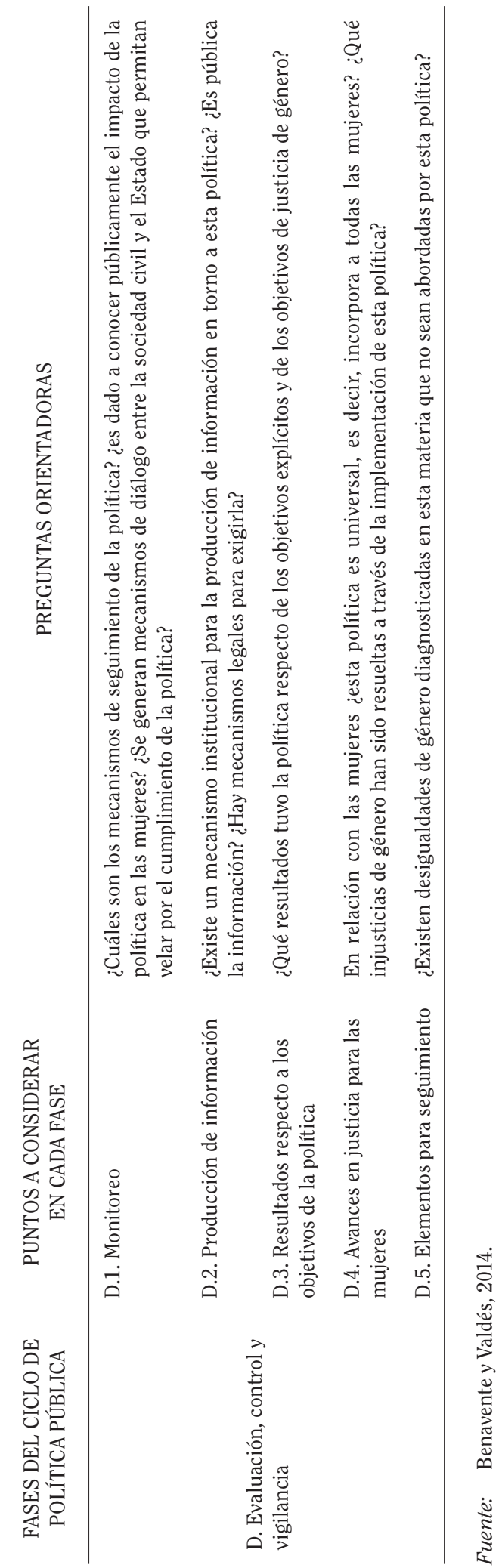

CC) (7) (8) Rev. Ciencias Sociales Universidad de Costa Rica, 165: 135-154 / 2019 (III). (ISSN: 0482-5276) 
Aplicada en el caso concreto de la PIGPJ, este instrumento orienta la sistematización y concentra el análisis en determinar su contenido, cómo se definió, en qué contexto, qué actores participaron de su construcción y puesta en marcha, así como, logros, obstáculos y nudos críticos. La reflexión propuesta considera el logro de objetivos de igualdad y justicia en el marco del rol que tienen las instituciones del sistema democrático en garantizar mecanismos para exigir los derechos formales $y$ transformar las relaciones de poder asentadas en la cultura. Lo novedoso de la aplicación a la PIGPJ, de esta matriz y del enfoque de justicia de género, es que permite examinar la capacidad de erradicar las injusticias que experimentan las mujeres en el campo jurídico y contribuir a la reivindicación de sus derechos humanos.

Para producir la información necesaria se realizó una revisión de los documentos oficiales de la PIGPJ, incluyendo la única evaluación existente, realizada en el año 2012 por la Dirección de Planificación del Poder Judicial. A estos insumos se les aplicó análisis de contenido, enfocado particularmente a identificar el significado que se atribuye a las desigualdades de género en el Poder Judicial, así como, al modo en que se produce el discurso institucional que procura contrarrestar las injusticias. El análisis de contenido se entiende como un conjunto de técnicas y la orientación teórica que las aglutina es el paradigma de justicia de género.

Además de la revisión documental, se realizaron entrevistas semiestructuradas con el fin de ahondar en aspectos que no se explicitan en los textos existentes. La entrevista se aplicó a 3 funcionarias judiciales, seleccionadas en función de que cumplen con los siguientes criterios indispensables para investigación: experticia y formación en género y políticas públicas, participación activa en el diseño e implementación de la PIGP y responsabilidad en la dirección de acciones contempladas en la política.

El objetivo de las entrevistas fue producir los insumos para reconstruir el contexto, actores institucionales y externos, intereses manifiestos $y$ tácitos, debates internos $y$ aquellas coyunturas que derivaron en la determinación del problema principal, en el planteamiento final, así como, sus logros y contradicciones. Para el análisis fue crucial contar con el criterio experto sobre la PIGP de las 3 mujeres entrevistadas:

1) Xinia Fernández Vargas es licenciada en Trabajo Social, especialista en derechos humanos de las mujeres $y$ violencia de pareja, doméstica y sexual. Es funcionaria judicial desde 1994, actualmente ocupa el cargo de Jefatura en la Secretaría Técnica de Género, instancia encargada de promover, orientar, fortalecer y monitorear los procesos de cambio tendientes a implementar la Política de Igualdad y Equidad de Género en la institución.

2) Eugenia Salazar Elizondo es máster en Derecho Penal. Labora en el Ministerio Público desde 1995; a partir del año 2008 $y$ hasta 2018 fue Fiscala Adjunta especializada contra la violencia de género, órgano con competencia nacional que tiene el propósito de mejorar la atención, trámite e investigación de los delitos derivados de este tipo de violencia. Actualmente, se desempeña como Fiscala Adjunta contra la Trata de Personas.

3) Milagro Rojas Espinoza es máster en Derecho y especialista en Género. Ha ejercido como magistrada suplente de la Sala Segunda. En la actualidad se desempeña como Jueza de Familia, Niñez y Adolescencia. Fue la primera persona en ocupar el cargo de Secretaria Técnica de Género y bajo su responsabilidad se diseñó la PIGPJ.

IV. ANÁLISIS DE LA POLÍTICA DE IGUALDAD DE GÉNERO DEL PODER JUDICIAL

A) IDENTIFICACIÓN Y DEFINICIÓN DE LOS PROBLEMAS PÚBLICOS A LOS QUE RESPONDE

\section{ANTECEDENTES Y ACTORES INVOLUCRADOS}

El contexto costarricense de la primera década del siglo xxı, se caracterizó por la profundización de las brechas de género derivada de 
procesos de empobrecimiento, desregulación del mercado laboral y precarización del empleo, relacionados con la persistencia de patrones culturales discriminatorios, tal es el caso de la segregación de género en la formación profesional, la violencia machista y la escasez de recursos públicos destinados a promover la equidad. Estas circunstancias incidieron en un avance muy lento de los esfuerzos estatales para erradicar la desigualdad de género (INAMU, 2007), reflejo de la contradicción que existe entre el reconocimiento jurídico de los derechos de las mujeres y el debilitamiento de la institucionalidad del Estado. Para Benavente y Valdés (2014) esta coyuntura en los países latinoamericanos guarda relación con una tendencia a desregular los mercados financieros y laborales privilegiando el crecimiento económico y la acumulación por encima de objetivos que promuevan la igualdad $y$ redistribución.

A partir de las entrevistas realizadas, se identifican como actores principales de la Política de Igualdad de Género del Poder Judicial: la Corte Plena, la Comisión de Género, la Secretaría Técnica de Género y el Instituto Nacional de las Mujeres como ente colaborativo. Fue impulsada a partir de dos precedentes, el primero es el Encuentro de Magistradas de América Latina $y$ el Caribe por una jurisprudencia con perspectiva de género, realizado en la ciudad de San José en el año 2001 y organizado por iniciativa de la entonces magistrada Zarella Villanueva Monge. Este espacio permitió articular esfuerzos y como principal producto tuvo una declaración que posteriormente fue presentada ante la Corte Plena. El 2 de abril del mismo año, la cúpula del gobierno judicial toma varios acuerdos, entre los más importantes: 1) transversalizar la perspectiva de género en los procesos de modernización y 2) crear una comisión integrada por representantes de las cuatro Salas de la Corte con el fin de "...diseñar una política integral que homogenice la perspectiva de género $y$ articule los servicios existentes para la atención de violaciones a derechos derivados de la condición de género de las personas..." (Poder Judicial, 2005, p.5).

Ambas acciones fueron incluidas en la propuesta para la segunda etapa del Programa de Modernización de la Administración de Justicia de Costa Rica. El Banco Interamericano de Desarrollo - principal financista de estas reformas en América Latina- solicitó con el interés manifiesto de que se cumpliera cabalmente la propuesta, que se operacionalizara con el diseño de una política y con la instauración de un órgano capaz de ejecutarla $y$ darle seguimiento (M. Rojas, comunicación personal, abril de 2018).

Como resultado de ese compromiso contractual con el BID, en el año 2002, inicia labores la Secretaría Técnica de Género y 3 años después, en noviembre de 2005, es aprobada la Política de Igualdad de Género del Poder Judicial, la cual se elaboró con apoyo del Instituto Nacional de las Mujeres mediante contratación de servicios profesionales externos. La etapa de diseño comprendió un proceso de consulta participativa a lo interno de la institución, una vez sistematizada, se efectuó una validación con personal judicial de distintas oficinas, tarea que en primera instancia estuvo a cargo de la Secretaría Técnica de Género pero que, con el segundo desembolso del Proyecto Corte$\mathrm{BID}$, recibe recursos económicos para llevarse a cabo por un equipo de especialistas a través de consultoría. Tal como señala Xinia Fernández (comunicación personal, febrero de 2018) no existían precedentes de una elaboración similar en otras instituciones de la República ni en otros Poderes Judiciales de la región.

\section{MARCO NORMATIVO}

La PIGPJ se basa en un marco jurídico nacional e internacional que tutela la igualdad, acceso a la justicia y el debido proceso. Sus principales referentes son la Constitución Política de Costa Rica, la Declaración Universal de Derechos Humanos, la Convención Americana de Derechos Humanos o Pacto de San José, Pacto Internacional de los Derechos Civiles y Políticos. Como marco legal específico destacan la Convención para la eliminación de todas las formas de discriminación contra la mujer conocida como CEDAW (1979); el Estado costarricense al suscribirla, se comprometió a proteger jurídicamente a las mujeres para alcanzar la igualdad de facto. 
Por su parte, la Plataforma de Acción de la Conferencia Mundial de la Mujer (1995) motiva a los países a revisar sus leyes y prácticas jurídicas para asegurar la revocación de las normativas que resulten discriminatorias y que obstaculicen la administración de justicia. Aunque no se incluyen instrumentos del derecho internacional en materia de violencia contra la mujer dentro del marco jurídico, contempla algunas acciones estratégicas, específicamente en la judicatura, donde se solicita aplicar dentro de las resoluciones, la Convención Interamericana para prevenir, sancionar y erradicar la violencia contra la mujer —Belem do Pará— de 1994.

El marco jurídico, según Benavente y Valdés (2014), permite la exigencia de derechos por parte de las personas destinatarias. En el caso de la PIGPJ, por su énfasis en la discriminación, el principal referente de justiciabilidad es la CEDAW (1979). La coyuntura social propició que la discriminación de género y no la violencia - como expresión concreta de este fenómeno- constituyera el núcleo de la propuesta. En el año 2005, aún no había sido aprobada en el país la Ley 8589 de Penalización de la Violencia contra las Mujeres, por lo cual una gran parte de estas agresiones no se judicializaba o lo hacía como violencia doméstica, categoría que oculta la direccionalidad y carácter estructural dentro de las relaciones familiares y de pareja. Esta situación además influyó en que no se articulara en el momento de su diseño con un plan gubernamental que delimite responsabilidades para las distintas instituciones, ya que también antecedió a la elaboración de la Política Nacional que coordina el INAMU para el decenio 2007-2017 con el objetivo de eliminar las brechas de género a nivel país.

\section{IDENTIFICACIÓN DEL PROBLEMA}

Desde el enfoque de justicia de género, la delimitación del problema debe hacer explícitos los efectos de la desigualdad en determinada población y las injusticias específicas, cuyo abordaje es el fin último (Benavente y Valdés, 2014). El problema que aborda la PIGPJ es la contradicción entre la igualdad formal y la desigualdad social que experimentan las mujeres, manifiesta en sus condiciones de vida $y$ oportunidades en relación con los hombres y el hecho de que el sistema judicial, lejos de mitigar dichas relaciones, ha contribuido a reproducirlas de diversas maneras; en el caso del Derecho, uno de los mecanismos es la falacia de la neutralidad valorativa $y$ de la igualdad como principio normativo (Poder Judicial, 2005). En este sentido, se apega a lo planteado por la CEDAW respecto a la pertinencia de concretar acciones que coadyuven a la equidad de género, sin embargo, la discriminación puede resultar en una serie de efectos concretos: en el acceso, interpretación y aplicación de la justicia, así como, obstáculos en el desempeño de las personas que laboran en la institución —que reciben los efectos de la desigualdad estructural y que los reproducen en sus funciones - los cuales no se clarifican en el planteamiento de objetivos.

\section{IDENTIFICACIÓN DE INJUSTICIAS DE GÉNERO}

Las injusticias específicas que viven las mujeres en el campo de la administración de justicia deben entenderse como resultado de la asimetría de poder $y$ de sus desigualdades situadas. La política se basó en un diagnóstico realizado en el año 2001 a manera de estudio exploratorio, que identificó necesidades y que orientó las líneas de acción, señalando 2 niveles de impacto: en el funcionamiento interno y a lo externo - entendido como las situaciones $y$ efectos sobre las mujeres que son usuarias de los servicios de la institución-. Sin embargo, la lectura que realiza de las injusticias es limitada, ya que pocas de las acciones planteadas se enfocan en problemas concretos.

En cuanto a las inequidades internas, menciona la ausencia de lenguaje inclusivo, nula incorporación de la perspectiva de género en reformas legales o propuestas de ley, dificultad en el acceso a incentivos $y$ puestos de toma de decisiones por parte de las funcionarias judiciales, masculinización de espacios como la policía, poca especialización del personal que tiene bajo su responsabilidad la atención de la violencia y delitos sexuales. Las injusticias identificadas a nivel externo son la ausencia de un enfoque de género y los prejuicios sexistas en las decisiones judiciales y en el abordaje de los casos. 


\section{B) FORMULACIÓN Y DISEÑO}

\section{OBJETIVO DE LA ACCIÓN ESTATAL}

El objetivo principal se planteó como "garantizar la igualdad de oportunidades entre mujeres $y$ hombres $y$ la no discriminación por género en las decisiones judiciales, en el servicio público de la administración de justicia, $y$ en el funcionamiento interno" (Poder Judicial, 2005, p. 9). Su planteamiento no cuenta con objetivos específicos, además el objetivo general y la meta son idénticos a pesar de que se trata de dos dimensiones distintas, pues las metas son generales mientras el objetivo debe ser una acotación del problema que se espera modificar. Ni en la declaración de intenciones ni en la definición del problema se posiciona a las mujeres como destinatarias y actoras en pro de la igualdad; de lo enunciado en documentos se infiere que ellas por su condición están en el núcleo del problema identificado; sin embargo, no se especifica el efecto esperado en su situación actual, tampoco se diferencian los distintos colectivos desde su particularidad (transexuales, afrodescendientes, indígenas, niñas, adolescentes, discapacitadas, adultas mayores, migrantes) que requieren una respuesta judicial prioritaria. A criterio de Xinia Fernández (comunicación personal, febrero de 2018), aunque a nivel enunciativo las mujeres no aparezcan como destinatarias, en la práctica el trabajo se ha concentrado en ellas, principalmente porque se introduce la perspectiva de género como una forma de visibilizar situaciones problemáticas, no surge de la identificación de un problema concreto o manifestación específica de la desigualdad y por esa razón, no particulariza una población meta, en su opinión este planteamiento general permitió contar con un margen de acción mayor para la función de la Secretaría Técnica de Género.

Los marcos de sentido otorgan un lugar preponderante al cumplimiento de directrices y normas establecidas de manera centralizada por el Gobierno Judicial, así lo confirman las 3 profesionales entrevistadas para este análisis. Actualmente, no se da seguimiento a la aplicación de los memorandos y circulares emitidos por los órganos superiores de la institución y no hay forma de garantizarse que quienes se incorporan a nuevos puestos conozcan la normativa interna (X. Fernández, comunicación personal, febrero de 2018). Este razonamiento coincide con lo que Winchester (2011) denomina paradigma de gestión burocrática tradicional, que se caracteriza por el control, la verticalidad y el logro de productos; ante la complejidad del contexto actual y sus demandas, esta forma de gerencia institucional es desplazada por nuevos modelos que enfatizan logros cualitativos respecto a las necesidades sociales, participación ciudadana y transparencia como pilares del quehacer de las instituciones públicas.

\section{MARCO POLÍTICO SOCIAL}

La participación de la sociedad civil dentro de la PIGPJ es inexistente, pues la formulación y el diseño estuvo a cargo de especialistas que efectuaron consulta $y$ validación con funcionarias $y$ funcionarios judiciales, pero no a nivel comunitario ni con grupos organizados. Además, el diálogo y la retroalimentación de otras instituciones se restringió a la brindada por el INAMU, institución con la que se coordina actualmente para la remisión de informes de cumplimiento de las Convenciones CEDAW y Belem do Pará (x. Fernández, comunicación personal, febrero de 2018). La implementación y el seguimiento a cargo de la Secretaría Técnica de Género tampoco ha incorporado los puntos de vista de organizaciones de mujeres, por lo que no es posible identificar consensos sociales ni elementos en disputa en su implementación, debido principalmente a que no se han abierto canales de comunicación y debate con otros actores. Para Benavente y Valdés (2014), el proceso deliberativo y participativo es fundamental en todas las etapas para incorporar las necesidades expresadas por las mujeres $y$ democratizar la gestión pública, lo que contribuye a la vigilancia en el cumplimiento de las acciones, pues tiene el efecto de generar compromisos $y$ capacidades colectivas e individuales para el reclamo y la denuncia, aporta herramientas para el diálogo y puede además abrir espacios de incidencia ciudadana en la toma de decisiones. 
La Comisión Nacional para el Mejoramiento de la Administración de Justicia (2015) afirma que la participación ciudadana, como deber y derecho en el Poder Judicial, es insuficiente debido a un conjunto de condiciones externas e internas; entre estas, la estructura $y$ naturaleza de la institución, la cultura judicial articulada por la verticalidad y jerarquía, limitaciones en el acceso a información relevante sobre las resoluciones judiciales y la necesidad de desvincular el sistema judicial de la sociedad como una forma de preservar un principio desvirtuado de independencia en la resolución del conflicto; el conjunto de estos elementos atenta contra una visión que coloca en su centro a las personas. Posterior a la formulación de la PIGPJ, en el año 2015, el Gobierno Judicial incorpora oficialmente la participación ciudadana como un recurso que contribuye a enriquecer y complejizar las acciones en distintos frentes.

\section{C) IMPLEMENTACIÓN}

\section{DESCRIPCIÓN GENERAL}

Esta sección analiza quién tiene la responsabilidad de la implementación, cuáles son las medidas para seguir un plan establecido, así como, la dinámica resultante en los ámbitos administrativos, políticos y sociales (Benavente y Valdés, 2014). La Comisión de Género se constituye en el ente director de esta iniciativa, la integran magistrados y magistradas, representantes de oficinas $y$ de asociaciones de servidores y servidoras judiciales. La implementación es responsabilidad de la Secretaría Técnica de Género, para lo cual, además de su personal permanente, ha establecido una estructura que descentraliza esta labor, con la creación de comités en los distintos circuitos judiciales del país, los cuales realizan acciones que se basan en el voluntariado de sus integrantes. Propone 5 áreas estratégicas que son: 1) la capacitación y sensibilización continua $y$ sistemática para todo el funcionariado, 2) la divulgación en medios internos y locales dirigida a todo el personal $y$ a las personas usuarias, 3) coordinación con otras instancias, 4) investigación, 5) seguimiento y evaluación. Estas áreas deberían emanar del campo jurídico constituido por las relaciones entre quienes aplican las leyes y el conjunto de la sociedad en general, enfatizando los efectos que la administración de justicia tiene sobre colectivos discriminados por el género.

La PIGPJ carece de un plan de acción, no delimita un plazo temporal para su implementación ni para el cumplimiento de las metas y acciones específicas, pues desde sus inicios se ha operativizado en los planes anuales de trabajo de la Secretaría Técnica de Género, los cuales se elaboran a partir de decisiones de la Comisión de Género, órgano de alto nivel que fija las líneas a seguir (X. Fernández, comunicación personal, febrero de 2018), esta imbricación con las labores de la Secretaría hace que sea difícil monitorear el avance que se supone debe ser progresivo $y$ constante.

Los mandatos de las Convenciones Internacionales como la CEDAw y Belém do Pará no se encuentran alineados con la planificación, lo que impide articular, sistematizar y reportar de manera sencilla el cumplimiento de las obligaciones asumidas por el país, ante el Comité CEDAW (X, Fernández, comunicación personal, febrero de 2018). Asimismo, la investigación de las brechas de género es un área poco desarrollada en la institución, ya que existen escasos recursos para ello, en este contexto las decisiones se basan en aspectos como la estadística o la incidencia de algunas situaciones, sin que existan estudios rigurosos sobre las relaciones desiguales de género y la forma en que las ha modificado la PIGP.

\section{TRANSVERSALIDAD}

Esta variable se refiere al involucramiento de los sectores, a los compromisos políticos y administrativos, al grado de formalización de acuerdos, así como, ajustes en los procedimientos de seguimiento, aplicación y evaluación (Benavente y Valdés, 2014). El Poder Judicial de Costa Rica se organiza en 3 ámbitos con funciones diferenciadas (administrativo, jurisdiccional y auxiliar de justicia), por lo cual, la PIGP establece acciones generales para cada ámbito. A criterio de Xinia Fernández (comunicación personal, febrero 
de 2018) un aspecto que contribuye a la institucionalización es que define 2 órganos de alto nivel como responsables de promover, orientar y monitorear su implementación. Para transversalizar se introduce como eje de los Planes Estratégicos Quinquenales, que son la base a partir de la que deben realizar la proyección del trabajo cada una de las oficinas.

La supervisión de los planes anuales operativos es responsabilidad de la Dirección de Planificación en coordinación con la Secretaría Técnica de Género. En promedio tres cuartas partes de las oficinas incluyen por lo menos una acción destinada a promover la equidad de género, pero en cuanto al cumplimiento se ha constatado que algunos temas (por ejemplo, infraestructura, diseño de edificios, protocolos de atención e investigación) son sujetos a una verificación acuciosa mientras otros no lo son, tal es el caso del seguimiento a las resoluciones judiciales para que sean inclusivas de la perspectiva de género (Dirección de Planificación, 2012).

\section{EVALUACIÓN}

La evaluación es un eje estratégico establecido en la política, pero en la práctica no se le ha concedido relevancia. Una de las mayores restricciones para analizar el efecto en la erradicación de las injusticias de género es que no se establecieron mecanismos de monitoreo ni indicadores de referencia vinculados al diagnóstico y diseño, como resultado, las evaluaciones que se realicen tendrán la limitante de no estar relacionadas con la propuesta programática inicial. El único ejercicio evaluativo institucional se realizó en el año 2012, por parte de la Dirección de Planificación y se enfocó en aspectos como el presupuesto asignado, cantidad de capacitaciones en la materia impartidas, acciones desarrolladas por la Secretaría Técnica de Género y percepción del funcionariado sobre las desigualdades, no explora el impacto en el acceso a la justicia de las mujeres, tampoco los efectos internos, por ejemplo, el acceso de las funcionarias a puestos de toma de decisiones o la erradicación del acoso sexual dentro de la institución. Por lo tanto, existe una carencia de mecanismos de evaluación concretos y periódicos, por medio de los cuales pueda evaluarse su impacto.

\section{INFORMACIÓN Y DIFUSIÓN}

Los actores institucionales y sociales conocen acerca de la implementación de la política por medio de campañas informativas, según está contemplado en el segundo de sus ejes estratégicos. Las plataformas electrónicas y audiovisuales son recursos utilizados para sensibilizar al funcionariado respecto a la violencia de género, delitos sexuales, lenguaje inclusivo $y$ no sexista, entre otros temas. Asimismo, se han dirigido también a público externo, principalmente a mujeres, ofreciendo información sobre la Ley de Paternidad Responsable, violencia intrafamiliar $y$ de género, delitos sexuales $y$ los mecanismos de denuncia. En el caso de la sociedad civil $y$ de instituciones públicas, esta labor se complementa con algunos talleres dirigidos a reforzar la educación en derechos. Pese a su importancia, estos espacios han sido escasos, debido principalmente a las limitaciones en recursos humanos y presupuestarios de la Secretaría Técnica de Género. En el año 2016, se consolidó el Observatorio de Género como un proyecto que aspira a mejorar y consolidar la información que se ofrece a distintos perfiles de personas, utilizando una plataforma virtual que actualiza en temáticas relacionadas.

\section{SOSTENIBILIDAD}

Según el único informe de evaluación (2012) no existe un parámetro de comparación para determinar la idoneidad de la inversión en materia de género en el Poder Judicial, lo que se explica por la ausencia de una línea de base. Ante la carencia de indicadores, la Secretaría Técnica de Género sugiere el análisis de la asignación presupuestaria de las oficinas responsables de materias sensibles (por ejemplo, Juzgados de Violencia y Pensiones Alimentarias, Familia, Fiscalía de Delitos Sexuales), si se suman los rubros de recurso humano y gasto variable de estas materias, la inversión representa el $11,77 \%$ del presupuesto total asignado para la institución en el año 2012 (Dirección de Planificación, 2012). 
Sin embargo, considerar la inversión presupuestaria asignada no es congruente con su propuesta programática, ya que los ejes estratégicos definidos no reflejan acciones concretas para la penalización de la violencia contra la mujer y no contrastan la eficacia de la asignación presupuestaria frente a las necesidades reales de la población. Otro parámetro es analizar la capacidad técnica y operativa de la Secretaría Técnica y la Comisión de Género como responsables de la implementación: el recurso humano asignado a la primera de estas instancias se ha incrementado considerablemente, si se compara con la dotación inicial del año 2005, sin embargo, su crecimiento se estancó, debido a la necesidad de restringir el gasto público y direccionar recursos a otras áreas del Poder Judicial. La estrategia de los Comités de Género ha procurado ampliar la cobertura en zonas fuera del Gran Área Metropolitana, pero sin contar con recursos asignados. En el caso de la Comisión de Género, instancia de alto nivel, no cuenta con presupuesto asignado para la concreción de acciones de seguimiento y funciona como recargo a las labores ordinarias de magistradas $y$ magistrados que la integran.

\section{DESARROLLO DE CAPACIDADES, COMPETENCIAS Y HABILIDADES}

La capacitación a funcionarias públicas $y$ funcionarios públicos para el desarrollo de habilidades y competencias es uno de los ejes estratégicos con más importancia, desde el punto de vista de la inversión de recursos para transformar las desigualdades de género en el ámbito de la cultura por medio de la andragogía. A pesar de que existen imprecisiones en el registro estadístico, desde su aprobación hasta el año 2012, se capacitaron alrededor de 7500 servidores $y$ servidoras judiciales principalmente en los módulos de "Perspectiva de Género y Accesibilidad, Derechos Humanos y Administración de Justicia", lo que representó en su momento al $75 \%$ de la planilla de personal (Dirección de Planificación, 2012).

Vargas y Nuñez (2012) concluyen que capacitar es una estrategia privilegiada dentro de la política pero que esta labor no se traduce en modelos integrados, permanentes y progresivos de formación. El Poder Judicial concentra instancias altamente especializadas y con distintas naturalezas funcionales, en consecuencia, el desarrollo de competencias debe atender a esa segmentación de las áreas de trabajo, pero procurando que la oferta formativa sea homogénea y siga directrices establecidas. El alcance de la capacitación difiere de un ámbito a otro, la mayoría se concentra en un nivel básico de sensibilización mientras que la especializada es esporádica, situación en la que influye el hecho de que la Secretaría no cuenta con personal suficiente para atender la totalidad de las demandas generadas de supervisión y acompañamiento, así como, el que las unidades de capacitación carecen de criterios de contenido y experticia necesaria en el tema (Vargas y Nuñez, 2012).

En el año 2011, la Secretaría Técnica de Género tuvo un acercamiento con las 5 unidades de capacitación para acotar parámetros sobre la inclusión de la perspectiva de género (Dirección de Planificación, 2012), a partir de entonces es posible advertir un cambio en el enfoque de las capacitaciones específicas, algunos ejemplos son los talleres sobre la investigación del femicidio dirigido a fiscales y personal de la policía judicial; la capacitación sobre el abordaje de los delitos sexuales según el protocolo de atención de las 72 horas dirigido a personal de la Oficina de Atención y Protección a la Víctima del Delito y los talleres sobre acoso sexual callejero dirigidos a personal judicial de distintas dependencias (E. Salazar, comunicación personal, febrero de 2018).

Ponderar los procesos de formación en términos de eficacia, es decir, respecto al impacto que tienen en incrementar el acceso a la justicia de las mujeres usuarias aún es tarea pendiente, pues no existe un sistema de indicadores cualitativos ni de evaluación para ello. En el nivel más básico, las capacidades adquiridas deberían reflejarse en un trato adecuado hacia las usuarias y en el grado de satisfacción de sus necesidades. Los informes de la Contraloría de Servicios del Poder Judicial (2016), oficina encargada de gestionar las inconformidades, revelan que durante el último quinquenio, las materias sensibles al género (tal es el caso de pensiones alimentarias y familia) acumulan 
un porcentaje significativo de quejas, debido principalmente a la ausencia de una resolución pronta y cumplida; en el caso de la atención de la violencia doméstica, muchas de las gestiones presentadas se refieren a trato inadecuado hacia las usuarias.

\section{D) EVALUACIÓN, CONTROL Y VIGILANCIA}

\section{RESULTADOS RESPECTO A LOS OBJETIVOS PROPUESTOS}

Corresponde sistematizar algunos de los resultados alcanzados respecto al objetivo de garantizar la igualdad de oportunidades y equidad de género, a nivel externo $y$ en el funcionamiento interno. Sobre la manera en que la PIGPJ ha impactado el servicio, cabe referirse a 2 aspectos, la articulación para la atención de violaciones a los derechos que deriven de la condición de género $y$ la respuesta que las mujeres reciben a sus demandas.

Previamente no existía un modelo de coordinación para facilitar la denuncia y el abordaje en delitos sexuales, violencia de género e intrafamiliar. A criterio de Xinia Fernández (comunicación personal, febrero de 2018) durante los últimos años, el Poder Judicial experimenta una transformación hacia una administración de justicia centrada en las personas, lo que implica un cambio cultural más que un cambio de procedimientos. Desde su punto de vista, la PIGP ha contribuido con un nuevo discurso y marco de referencia, $y$ a nivel general sentó las bases para sensibilizar sobre la existencia de las desigualdades de género y procurar servicios cada vez más especializados. Reflejo de ello es la creación de la Fiscalía Especializada de Género y Delitos Sexuales, la Comisión de Seguimiento a la Violencia Intrafamiliar, así como, la Comisión de Seguimiento a la Ley de Penalización de la Violencia
Contra las Mujeres, todas como acuerdo de la Comisión de Género.

Uno de los productos destacados en cuanto a proyección e inversión, es la Plataforma Integrada de Servicios para la Atención de las Víctimas (PISAV) creada en el año 2011, con el fin de agilizar una respuesta judicial para esta población. Integra al Juzgado de Violencia Doméstica, Juzgado de Pensiones Alimentarias, Fiscalía, Defensa Pública, Departamento de Trabajo Social y Psicología, Oficina de Atención y Protección a la Víctima del Delito y Unidad de Medicina Legal. En un solo lugar, las personas denunciantes de violencia pueden además iniciar procesos de demanda por alimentos, tener asistencia legal gratuita y también atención psicosocial.

La estructura de PISAV aportó 2 cambios significativos: la unificación y concentración de servicios en sitios con una alta demanda en las materias señaladas, así como, el enfoque especializado del personal a cargo. Asimismo, procura facilidades para la atención inmediata a víctimas de delitos de violencia sexual, bajo el esquema de intervención única, tal es el caso de toma de denuncia, medicina legal y asistencia médica. No es posible referirse al impacto de PISAV, ya que no existen mecanismos de evaluación y monitoreo acordes a la realidad $y$ necesidades de las mujeres, pero según datos estadísticos, la plataforma tiene un impacto en las intervenciones judiciales relacionadas con penalización de la violencia, específicamente en la reducción de las solicitudes de desestimación $y$ sobreseimiento si se compara con el promedio nacional (cuadro 2).

La desestimación es una forma de cierre del proceso antes de llegar a la etapa de juicio, se formula ante alguna de las siguientes circunstancias: i) imposibilidad de proseguir con la investigación, ii) los hechos no constituyen delito, o iii) los elementos de prueba son insuficientes para fundamentar una acusación (Código Procesal Penal, art. 282, 1998). 
CUADRO 2

SOLICITUD DE DESESTIMACIONES Y SOBRESEIMIENTOS

COMPARACIÓN MODALIDAD PISAV-PROMEDIO NACIONAL PERIODO 2015

\begin{tabular}{c|c|c}
\hline \multirow{2}{*}{ FISCALÍA } & \multicolumn{2}{|c}{ CASOS TERMINADOS (DENUNCIA DIRECTA) } \\
\cline { 2 - 3 } & DESESTIMACIONES & SOBRESEIMIENTOS \\
\hline PISAVPavas & $61 \%$ & $10 \%$ \\
PISAV La Unión & $45 \%$ & $7 \%$ \\
Porcentaje nacional & $76 \%$ & $13 \%$ \\
\hline
\end{tabular}

Fuente: Dirección de Planificación, 2015. Informe estadístico Fiscalías modalidad PISAV.

La respuesta que las mujeres reciben a sus demandas es el segundo aspecto sustancial. Desde la perspectiva de justicia de género, las políticas públicas deben transformar las relaciones de desigualdad ofreciendo una respuesta a las necesidades históricamente invisibilizadas. Es insuficiente la información producida por la institución, así como, la generada desde espacios como la academia y las organizaciones de sociedad civil para auditar la calidad de la administración de justicia y dimensionar la discriminación de género en las decisiones judiciales.

Un indicador indirecto es la tutela efectiva de derechos o "juicio justo", que se entiende como el libre acceso al sistema de administración de justicia para obtener una resolución de fondo por parte de la autoridad competente, ajustada al marco legal vigente. Esta dimensión es propuesta por el Primer Informe Estado de la Justicia para contribuir a supervisar el desempeño del Poder Judicial: permite identificar situaciones concretas en las que hay un riesgo en la protección de los derechos por vía judicial relacionado al perfil de las personas involucradas y según tipos de delito. Quienes denuncian delitos sexuales e infracciones a la Ley 8589 de Penalización de la Violencia contra las Mujeres, tienen una alta probabilidad de que sus derechos no sean efectivamente tutelados, en ambas categorías la posibilidad de que el caso sea desestimado alcanza el 80\% (Programa Estado de la Nación, 2015).

En este tipo de respuesta tiene un lugar preponderante la cultura que naturaliza la violencia hacia las mujeres, en contra de la protección que establece la normativa nacional e internacional vigente. Muchos de los casos se desestiman bajo el argumento de que la víctima no desea continuar con el proceso judicial, a pesar de que la Ley de Penalización 8589 en el art. 4 indica que todos los delitos que contempla serán de acción pública, es decir, el hecho de que se retire la denuncia no implica archivar el procedimiento, pues una vez iniciado, es obligación de la autoridad proseguir, agotando todos los elementos de prueba posibles. La investigación probatoria con perspectiva de género es un área poco desarrollada y dentro de los procesos que llevan a cabo la Fiscalía y el Organismo de Investigación Judicial, continúan presentándose prácticas que revictimizan a las mujeres y a sus familias. Esta situación claramente no ocurre con otros delitos, por ejemplo, con el narcotráfico, en donde hay una actitud mucho más proactiva de agotar todas las instancias, lo que no se hace con los delitos de LPVCM. Hay una construcción social que está vinculada a esa visión que se tiene de las mujeres como colectivo (x. Fernández, comunicación personal, febrero de 2018).

Lo señalado por el Estado de la Justicia en el año 2015, incidió en que el Gobierno Judicial y jefaturas prestaran mayor atención a las desestimaciones asociadas a delitos de violencia sexual $y$ procurasen acciones para disminuirlas, entre estas, el fortalecimiento de las unidades de control en el Ministerio Público y el registro ampliado de causales de desestimación desagregando 
la variable insuficiencia probatoria en 4 rubros a partir de la Circular 04 de 2016 del Ministerio Público (E. Salazar, comunicación personal, febrero de 2018); así como, la ampliación del Programa de equipos de respuesta rápida para la atención integral a víctimas de violación y la aprobación en septiembre de 2016 por parte del Consejo Superior del Plan de acción para la aplicación efectiva de la Ley de Penalización de la Violencia contra las Mujeres, sobre estos programas y planes no existen datos consolidados respecto al impacto (Programa Estado de la Nación, 2017).

Desde la perspectiva de Xinia Fernández (comunicación personal, febrero de 2018), la política ha permitido identificar nuevas necesidades de las usuarias no contempladas inicialmente, una de las más relevantes es la ausencia de representación legal gratuita para mantenerse en un proceso judicial que es complejo y revictimizante (aunque existan políticas). La Comisión de Género y el despacho de la expresidenta de la Corte Suprema, Zarella Villanueva, impulsaron en el año 2016, una propuesta de proyecto de ley para ampliar la representación legal de las víctimas y otorgarle rango constitucional.

También, la política se comprometió a erradicar la discriminación de género a lo interno del Poder Judicial, implementando las acciones afirmativas necesarias, pero tal como señala la Dirección de Planificación (2012), constituye un espacio donde persisten injusticias que impiden la igualdad de resultados, principalmente en las oportunidades de acceso de las mujeres a puestos de jefatura y a capacitaciones. No se han desarrollado medidas especiales de carácter temporal, integradas en una estrategia para corregir la representación insuficiente de la mujer y redistribuir los recursos y el poder (Comité cedaw, 1999), lo que se refleja en los datos más recientes respecto a los nombramientos en propiedad en puestos de alta jerarquía (cuadro 3 ).

CUADRO 3

DISTRIBUCIÓN DE PUESTOS DE ALTA JERARQUÍA EN EL PODER JUDICIAL JULIO 2018

\begin{tabular}{lcc}
\hline NOMBRE DEL PUESTO & CANTIDAD DE MUJERES & CANTIDAD DE HOMBRES \\
\hline Magistrado & 5 & 9 \\
Magistrado presidente de Sala & 1 & 3 \\
Integrante del Consejo Superior & 0 & 51 \\
Juez 5 & 34 & 174 \\
Juez 4 & 109 & 1 \\
Director General OIJ & 0 & 0 \\
Fiscal General & 1 & 8 \\
Administrador Regional 3 & 6 & 1 \\
Jefe de Prensa & 0 & 1 \\
Jefe Oficina de Planes y Operaciones & 0 & 0 \\
Jefe de la Defensa Pública & 1 & 251 \\
\hline Total & 158 & 2 \\
\hline
\end{tabular}

Fuente: Elaboración propia a partir de Estadísticas del Observatorio de Género del Poder Judicial, 2018.

Nota: $\quad$ El cuadro únicamente recupera el dato de los nombramientos en propiedad a julio de 2018. 
Desde la perspectiva de las 3 funcionarias entrevistadas, algunos de los principales logros promovidos por la PIGPJ a nivel interno son: la reforma al Reglamento de Hostigamiento Sexual en el empleo para que las mujeres afectadas figuren como parte en el proceso, ya que antes eran únicamente la instancia empleadora y el acusado; la generación de estadísticas con perspectiva de género que son la base para rendir informes de cumplimiento de la CEDAW; introducir la perspectiva de género dentro de los planes de capacitación $y$ generar un discurso institucional contra la discriminación de este tipo.

\section{CONCLUSIONES Y RECOMENDACIONES}

El análisis revela que la Política de Igualdad de Género del Poder Judicial fue diseñada $y$ aprobada en una década de estancamiento de los esfuerzos estatales para erradicar la desigualdad de género, en ese momento no existían precedentes de una elaboración similar en otras instituciones de la República ni en otros Poderes Judiciales de la región. Constituye un proyecto de emancipación que aspira a promover cambios en las condiciones de vida de las mujeres eliminando el desbalance de poder $y$ desventajas que el sistema patriarcal les asigna, además ha contribuido a problematizar la neutralidad del derecho $y$ generar un discurso institucional de reconocimiento de las desigualdades.

Encuentra sustento en un marco legal muy amplio desde el cual debería promoverse una mayor participación ciudadana para que las reivindicaciones se desarrollen a partir de debates y espacios de discusión que especifiquen los contenidos de la representación, la delegación de poder y el liderazgo de las mujeres para garantizar su empoderamiento y autonomía. En esta tesitura, el análisis revela que la política responde a un paradigma burocrático tradicional, caracterizado por la verticalidad y la jerarquía, generando un doble efecto: garantiza una estructura institucional para la implementación de un esfuerzo de este tipo a la vez que despolitiza e inhibe el logro de consensos con organizaciones ciudadanas y otros actores. Una de las áreas más desarrolladas es la articulación para la atención de violaciones a los derechos que deriven de la condición de género, muestra de ello es la creación de la Plataforma PISAV, que reúne bajo el esquema de intervención única, a una serie de oficinas judiciales que son requeridas en casos de violencia de género y que ha sido diseñada para facilitar la denuncia $y$ disminuir la revictimización en los procesos judiciales.

Una de las limitaciones de la PIGP es que no delimita un plazo temporal para su implementación ni para el cumplimiento de las metas y acciones; asimismo, carece de un plan de acción específico y de mecanismos de evaluación, monitoreo e indicadores de referencia. A 12 años de su aprobación requiere ser evaluada respecto al impacto que ha tenido para las mujeres que buscan acceder al sistema de justicia. Las políticas públicas tienen un ciclo $y$ una continuidad, lo que significa que no son estáticas y aun cuando logren transformaciones importantes, existirán nuevos problemas y situaciones por resolver, que ameritan un seguimiento constante $y$ en algunos casos redefinición para que sean eficaces frente a los nuevos desafíos (Benavente y Valdés, 2014).

La discriminación de género en las decisiones judiciales es un problema que persiste y cuyo análisis es necesario para auditar la calidad de la administración de justicia. Estos indicadores indirectos muestran que los delitos de violencia de género cometidos contra mujeres presentan una serie de condiciones fácticas que atentan contra el derecho a un "juicio justo", entre otras, deficiencias en la atención de las mujeres usuarias, retardo judicial, carencia de herramientas investigativas $y$ probatorias con perspectiva de género; así como, ausencia de habilidades y especialización en el recurso humano destinado a estos fines. Un diagnóstico amplio de los problemas más acuciantes puede tomar como punto de partida los que se mencionan, aunque posiblemente identifique otras situaciones que deben tenerse en consideración $y$ que no fueron contempladas dentro de los objetivos de la PIGPJ.

Después de evaluar y de diagnosticar la situación actual es recomendable que se diseñe una nueva política de género que armonice e 
integre la legislación vigente y los instrumentos internacionales, asimismo, se recomienda que esta política se integre o articule - especialmente en lo que respecta al abordaje de la violencia y su judicialización- con la Política Nacional de Igualdad y Equidad de Género que es coordinada desde el Instituto Nacional de las Mujeres. El nuevo diseño debe precisar los problemas concretos que se espera abordar en el campo jurídico en las dimensiones de acceso, interpretación y aplicación de la justicia; contemplar la participación de la sociedad civil y la corresponsabilidad de la ciudadanía para el cumplimiento de acciones; articular la capacitación dentro de un proceso formativo especializado de acuerdo a las áreas de trabajo con aplicación práctica; incorporar la interseccionalidad de la condición de género con la clase, etnia, religión, edad, orientación sexual y discapacidad; dirigir acciones específicas a los distintos colectivos de mujeres; así como, aplicar la justicia de género en los delitos de violencia para garantizar la tutela de los derechos. Finalmente, junto con el diseño debe elaborarse una línea de base y un sistema de indicadores que permitan monitorear los avances en aspectos como la selección de personal en puestos de jefaturas y jerárquicos, evaluar la plataforma PISAV e incluir criterios respecto al juicio justo.

Algunas de las líneas de investigación que este trabajo ha planteado sin desarrollar, por exceder su alcance, $y$ que pueden constituir materia para futuras reflexiones son la necesidad de profundizar en los mecanismos para el seguimiento por parte de la ciudadanía y la academia de iniciativas para auditar la calidad de la justicia que reciben las mujeres, desde el área de la evaluación de programas y políticas públicas. Este es un tema que se encuentra actualmente poco desarrollado, según pudo advertirse en el estado de la cuestión y que puede aportar posibilidades de incidencia $y$ de teorización importantes para el feminismo. Asimismo, es necesario reflexionar sobre las implicaciones que en el eventual diseño e implementación de una nueva política de género puede tener la reforma fiscal aprobada en el año 2018 y que supone, según criterio de la Corte Plena, una afectación en el funcionamiento del Poder Judicial, entre otras cuestiones, por las restricciones presupuestarias que conlleva, ante este panorama es preciso considerar posibilidades innovadoras de colaboración y de búsqueda de recursos externos que permitan hacer frente a las injusticias y lograr equidad con mayor participación de la sociedad civil.

\section{REFERENCIAS}

Benavente, M.C. y Valdés, A. (2014). Políticas públicas para la igualdad de género: $u n$ aporte a la autonomía de las mujeres. Libros de la CEPAL 130. Santiago, Chile: Comisión Económica para América Latina y el Caribe (CEPAL).

Birgin, H. y Gherardi, N. (2008). El acceso a la justicia como un derecho humano fundamental:retos $y$ oportunidades para mejorar el ejercicio de los derechos de las mujeres. En Etchegoyen, A. (Coord.), Mujer y Acceso a la justicia. Buenos Aires: El MonoArmado.1era edición. Recuperado de http://www.jusformosa.gob.ar/escuela/violencia/MOD7-3BibliografiaSugerida.pdf

Bourdieu, P. (2002). La fuerza del derecho. Bogotá, Colombia: Universidad de los Andes.

Carcedo, A. (2002). Propuesta de un sistema de indicadores para Costa Rica relativos a laviolencia doméstica y la violencia sexual contra las mujeres (Tesis para optar al grado de Magíster Scientiae). Universidad de Costa Rica, Programa de Estudios de Posgrado en Estudios de la Mujer, Costa Rica.

Castañeda, P. (2012). Epistemología feminista $y$ estudios sobre violencia de género: apuntespara la reflexión. En Ravelo, P. y Dominguez, H. (Coordinadores), Diálogos Interdisciplinarios sobre Violencia Sexual, (30-45). México: EON/ FONCA/CONACULTA.

Código Procesal Penal de Costa Rica (1998). Art. 282. Desestimación. Diario Oficial La Gaceta 106 del 04/06/1996. Recuperado de http://www.pgrweb.go.cr/ scij/Busqueda/Normativa/Normas/nrm_ norma 
Comité CEDAW. (1999). Recomendación general nro. 25, sobre el párrafo 1 del artículo 4 de la Convención sobre la eliminación de todas las formas de discriminación contra la mujer referente a medidas especiales de carácter temporal. Organización de Naciones Unidas. Recuperado de http://www.un.org/womenwatch/daw/ cedaw/recommendations/General $\% 20$ recommendation $\% 2025 \% 20$ (Spanish).pdf

Comité CEDAW. (2015). Recomendación General nro. 33 sobre el Acceso de las Mujeres a la Justicia. Organización de Naciones Unidas. Recuperado de http:// www.acnur.org/fileadmin/scripts/ doc.phpfile=fileadmin/Documentos/ BDL/2016/10710

Comisión Nacional para el Mejoramiento de la Administración de Justicia (CONAMAJ). (2015). Política de Participación Ciudadana en el Poder Judicial. Costa Rica.

Contraloría de Servicios del Poder Judicial (2016). Informes Estadísticos y de Logros Obtenidos. Costa Rica. Recuperado de https://contraloria.poder-judicial.go.cr/ documentos/informe-de-labores.

Convención Interamericana para Prevenir, Sancionar y Erradicar la Violencia contra la Mujer, Belém do Pará. (1995). Vigésimo Cuarto Periodo Ordinario de Sesiones. Organización de Estados Americanos. Recuperado de https://www. oas.org/dil/esp/convencion_belem_do_ para.pdf

Convención sobre la Eliminación de Todas las Formas de Discriminación contra la Mujer (CEDAW). (1979). Organización de Naciones Unidas, Comisión de la Condición Jurídica y Social de la Mujer. Recuperado de http://www.un.org/ womenwatch/daw/cedaw/text/sconvention.htm

Dirección de Planificación del Poder Judicial. (2015). Informe estadístico sobre Fiscalías modalidad PISAV. Costa Rica.

Dirección de Planificación del Poder Judicial. (2012). Evaluación de la Política de Género del Poder Judicial de Costa Rica. Costa Rica.
Facio, A. (1999). Feminismo, género y patriarcado. Revista sobre enseñanza del derecho de Buenos Aires, (6), año 3, pp. 259-294.

Facio, A. y Fries, L. (2005). Feminismo, género $y$ patriarcado. Revista sobre enseñanza del derecho de Buenos Aires, (6), 259294. Recuperado de http://www.derecho.uba.ar/publicaciones/rev_academia/ revistas/06/feminismo-genero-y-patriarcado.pdf

Instituto Nacional de las Mujeres. (2007). Politica Nacional para la Igualdad y Equidad de Género 2007-2017, 2. ed. Costa Rica.

Lagarde, M. (1998). Identidad genérica y feminismo. Sevilla, España: Instituto Andaluz de la Mujer.

Lahera, E. (2002). Introducción a las politicas públicas. Santiago, Chile: Fondo de Cultura Económica.

Ley 8589. Penalización de la Violencia contra las Mujeres. (2007). Diario Oficial La Gaceta nro.103 del 30/05/2007. Recuperado de http://www.pgrweb.go.cr/ scij/Busqueda/Normativa/Normas/nrm norma.aspx?param1=NRM\&nValor1=1\&n Valor2 $=60183 \& n$

Observatorio de Violencia de Género contra las Mujeres y Acceso a la Justicia. (2018). Distribución del personal por puestos según género. Recuperado de http:// www.poder-judicial.go.cr/observatoriodegenero/programas-acciones/comopatrono/distribucion-de-personal/

Pateman, C. (1955). El Contrato Sexual. Introducción de María Xosé Agra Romero. Barcelona: Editorial Antrophos.

Poder Judicial. (2005). Política de Igualdad de Género. Costa Rica. Recuperado de http://observatoriodegenero.poder-judicial.go.cr/wp-content/uploads/2018/02/ Politica-igualdad-genero-PJ.pdf

Programa Estado de la Nación. (2015). Primer Informe Estado de la Justicia. Costa Rica. Recuperado de http://www.estadonacion.or.cr/justicia/assets/estado-de-lajusticia-1-baja.pdf

Programa Estado de la Nación. (2017). Segundo Informe Estado de la Justicia. Costa Rica. 
Recuperado de https://www.estadonacion.or.cr/files/biblioteca_virtual/justicia/COMPLETO-2017.pdf

Reglas de Brasilia sobre Acceso a la Justicia de las Personas en Condición de Vulnerabilidad. (2008). Aprobadas por la XIV Cumbre Judicial Iberoamericana en Brasilia 4/3/2008. Recuperado de http:// www.acnur.org/fileadmin/Documentos/ BDL/2009/7037.pdf

Segato, R. (2003). Las estructuras elementales de la violencia. Bernal, Argentina: Universidad Nacional de Quilmes.

Trubek, D. y Dezalay, Y. (1997). La internacionalización de los campos jurídicos y la creación de espacios transnacionales. Pensamiento Jurídico, (1), 5-41. Colombia: Universidad Nacional. Recuperado de https://revistas.unal.edu.co/index.php/peju/article/ view/38887/pdf_288

Vargas, A. y Núñez, M. (2012). El Rol de la Capacitación Institucional en la
Incorporación de la Perspectiva de Género y la Politica de Equidad de Género del Poder Judicial Costarricense. (Tesis para optar por el grado de Licenciatura en Trabajo Social).Universidad de Costa Rica, Sede Rodrigo Facio.

Winchester, L. (14-25 de noviembre de 2011). La formulación e implementación de las políticas públicas en América Latina $y$ el Caribe. ILPES. Curso Internacional Políticas presupuestarias y gestión por resultados. Santiago: Chile. Recuperado de https://repositorio.cepal.org/bitstream/handle/11362/37223/S1420739_ es.pdf.txt

Fecha de ingreso: 18/09/2018 Fecha de aprobación: 15/07/2019 